\title{
Modeling of Magnetic Properties of GO Electrical Steel Based on Epstein Combination and Loss Data Weighted Processing
}

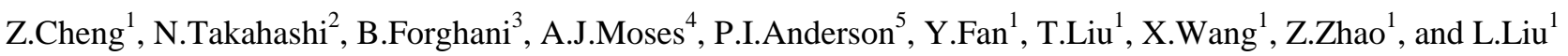 \\ 1 R \& D Center, Baoding Tianwei Group Co., Ltd, CHINA, email: emlabzcheng@yahoo.com \\ 2 Dept. of E.E., Okayama University, JAPAN \\ 3 Infolytica Corporation, CANADA, email: forghani@infolytica.com \\ 4 Emeritus Professor, Wolfson Centre for Magnetics, Cardiff University, UK, email: mosesaj@ cardiff.ac.uk \\ 5 Wolfson Centre for Magnetics, School of Engineering, Cardiff University, UK, email: andersonPI1@cf.ac.uk
}

\begin{abstract}
The extended modeling of the magnetic properties of GO (Grain Oriented) electrical steel is presented in this paper which is based on a set of standard and scaled-down Epstein frames and a proposed two-level weighted processing of Epstein data, including the mean magnetic path length, specific magnetization loss and exciting power. The effects of excitation frequency, strip angle and ambient temperature on the results obtained from the Epstein frames are investigated. It is shown that using the proposed Epstein combination and the two-level weighted processing method is an efficient way of building a model for determining magnetic losses more realistically, hence, improving the value of Epstein strip measurement data.
\end{abstract}

Index Terms - Epstein test, exciting power, grain oriented electrical steel, mean magnetic path length, specific magnetization loss.

\section{INTRODUCTION}

I MPROVED MODELS of the vector properties of localized flux density and the magnetic field, in laminations of grain oriented (GO) electrical steels assembled in energy efficient magnetic cores, are needed in order to predict the efficiency of electrical machines more accurately and/or to provide basic data to assist in developing enhanced core topologies. A clearer understanding of vector magnetic properties has led to improvements in basic measurement techniques and advances in material modeling methods [1-7], which in turn have led to significant progress in the design of efficient electromagnetic devices in recent years.

In spite of this progress basic scalar magnetic characteristics measured by means of either the Epstein frame or the single sheet tester (SST) [8-11] are still being widely used in industrial applications, although the rapid and extreme increases in voltage and ratings of electrical equipment worldwide makes the requirement for accurate magnetic property modeling more critical. In these cases, effects such as the additional iron loss in the external layer of the stacked core, induced by the leakage magnetic flux perpendicular to the laminations [12], or the high acoustic noise level, caused by DC-biasing in large power transformers [13], involving 3-D excitation, high frequency and high operating flux density [14], PWM (pulse width modulation) excitation [15], or other extreme working conditions [16] must be taken into account. Unfortunately, magnetic properties of GO electrical steel, measured under these non-standard conditions, are not widely available and rarely used in current commercial designs or performance predictions. The impact of these effects is recognized by manufacturers and users. However, despite publication of reports such as IEC/TR 62383 [17] which recognize the importance of measuring harmonic rich waveforms, little concern is given to the additional measurement errors introduced by the changes in the physical magnetization processes.

Manuscript received March 8, 2013, revised May 23, and June 29, 2013.

Corresponding authors: Z.Cheng (e-mail: emlabzcheng@yahoo.com).

Digital Object Identifier inserted by IEEE.
As in a single phase transformer core, the magnetic field, flux density, loss and exciting power, in laminations in the Epstein frame, are only uniform within the central portion of each limb. To attempt to compensate for this, the magnetic property data provided by the standard $(25 \mathrm{~cm})$ Epstein frame is effectively obtained by averaging, using the mean magnetic path length of $0.94 \mathrm{~m}$, according to the relevant IEC Standard [10]. However, it is commonly known that this value is not constant and using a fixed value can lead to significant errors, particularly when comparing losses or permeability of different grades of material, measuring under non-standard magnetizing frequencies or flux densities or, comparing measurements on Epstein strips cut at different angles to the RD (rolling direction) [16, 18-19]. Consequently, it is necessary to reexamine the traditional magnetic measurement methods and attempt to improve the processing of the measurement data in order to qualify the material property data frequently used $[12,19-20]$, and to verify, strictly, the modeling and simulation through well-established benchmarking problems [22-23].

This paper covers the following themes: (i) it shows the benefit of establishing an Epstein set, combining one standard frame $(25 \mathrm{~cm})$ and two scaled-down frames $(17.5 \mathrm{~cm}$ and 20 $\mathrm{cm})$, referred simply to as E-25, E-17.5 and E-20, into two Epstein groups, 2E(25-17.5) and 2E(25-20), respectively; (ii) it demonstrates the use of a weighted processing method, proposed by the authors, which is based on loss data and can be applied to reasonably determine the mean magnetic path length of the Epstein frame under various conditions; (iii) it examines the effect of the grade and texture of GO electrical steel, flux density, magnetizing frequency, ambient temperature, and the angle at which Epstein strips are cut to the RD, on the specific magnetization loss and exciting power (or specific apparent power).

\section{Methodology AND EPSTEIN SET}

The application-oriented improvement and re-examination of the traditional Epstein experiments was carried out with the 
aid of an Epstein set, model TYU-2000M, manufactured by Tianyu Electronics, Ltd, shown in Fig.1. This set comprises a standard Epstein frame (E-25) and two scaled-down Epstein frames, used for testing $20 \mathrm{~cm}$ and $17.5 \mathrm{~cm}$ strip lengths referred to as E-20 and E-17.5 respectively. An air compensation system is incorporated. The repeatability of all loss and exciting power measurement in each configuration was better than $\pm 1 \%$.

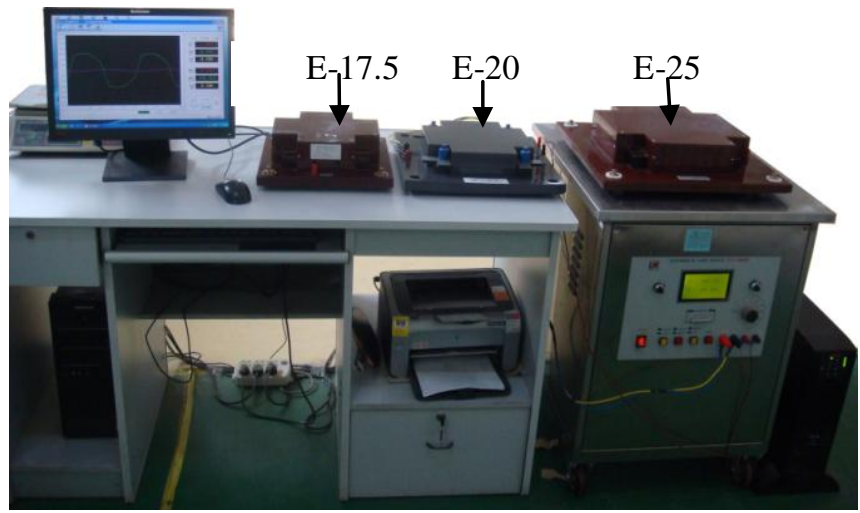

Fig.1. Epstein set showing E-25, E-20 and E-17.5 frames.

\section{A. Double Epstein Frame Method}

The double Epstein frame method reported in $[16,18]$ is intended to remove the effect of the non-uniform fields over the Epstein corner regions and to enable the accurate measurement of specific magnetization loss and exciting power in the uniformly magnetized regions of the limb. Two assumptions are made: (i) the non-uniform magnetic field distribution over the corner regions of both the standard Epstein frame $(25 \mathrm{~cm})$ and the scaled-down Epstein frame $(17.5 \mathrm{~cm}$ or $20 \mathrm{~cm})$, are identical, despite the difference in the size of the limbs in the two frames (see Fig.2(a) for E-25 and Fig.2.(b) for E-17.5); (ii) the magnetic field is uniform over the middle section of each limb as indicated in Fig.2(a).

In order to demonstrate the trend in the magnetic field distribution in the corner regions of the cores assembled from strips (grade: $30 \mathrm{Q} 120$, strip angle: $0^{\circ}$ ) of E-25 and E-17.5, 3-D magnetic field analysis was carried out using MagNet (Infolytica). Fig.2 shows that the magnetic field distribution in the non-uniform regions of the E-25 core, indicated in Fig.2(a), and all the non-uniform regions of E-17.5 or E-20 cores, shown in Fig.2(b), are identical, as assumed earlier. If the uniform regions, shown in Fig.2(a), are areas that are removed in the scaled-down Epstein frame (E-17.5 or E-20), then the sketches in Fig. 2 illustrate a "subtraction" process between results from the standard and the scalded-down Epstein frames.

\section{B. Two-Level Weighted Processing of Epstein Parameters}

A two-level weighted processing method is used in order to obtain a closer approximation for the mean magnetic path length of the standard Epstein frame (E-25), considering the non-uniform specific magnetization losses over the different regions, and to examine the effect of different Epstein groups on the magnetic properties.

\section{1) First-Level Weighted Processing}

The mean magnetic path length, $l_{m}$, of the standard Epstein frame is given by [18],

$$
l_{m}=4 l \cdot \frac{P_{n}}{m_{t} P_{\text {loss }}}
$$

where $l[\mathrm{~m}]:$ the total length of each Epstein strip; $P_{n}[\mathrm{~W}]$ : the absolute total magnetization loss of the standard Epstein frame (E-25); $m_{t}[\mathrm{~kg}]$ : the total mass of all the laminations inside the frame; $P_{\text {loss }}[\mathrm{W} / \mathrm{kg}]$ : the specific magnetization loss.
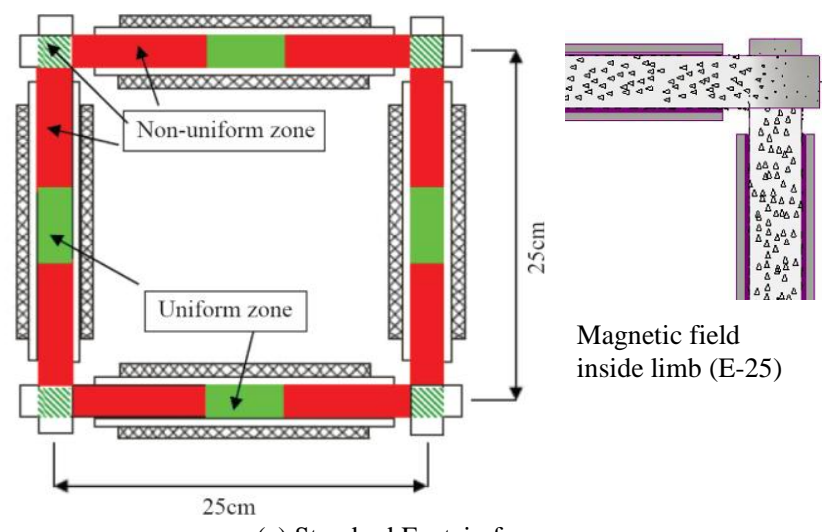

Magnetic field inside limb (E-25)

(a) Standard Epstein frame
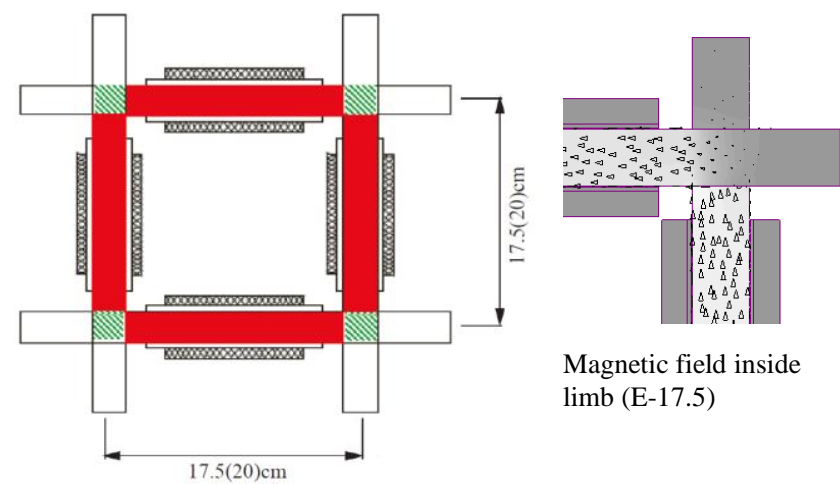

Magnetic field inside limb (E-17.5)

(b) Scaled-down Epstein frame (E-17.5 or E-20)

Fig.2. Schematic plan view of the Epstein subtraction model.

The mean magnetic path length, $l_{m}$, is dependent on the specific magnetization losses as shown in (1). However, the specific magnetization losses in the uniform and non-uniform zones of the entire Epstein frame (denoted by $P_{\text {loss } 1}$ and $P_{\text {loss } 2 \text {, }}$ respectively) differ due to the different field distributions illustrated in Fig.2. Therefore, two forms of mean magnetic path lengths, $l_{m l}$ and $l_{m 2}$, for the standard Epstein frame, can be determined based on the specific magnetization losses, $P_{\text {loss }}$ and $P_{\text {loss } 2}$, of the uniform and non-uniform sub-regions.

The specific magnetization loss over the indicated uniform zones of the Epstein limbs (see Fig.2(a)), $P_{\text {lossl }}$, can be determined from the difference between the absolute power losses obtained from the standard and the scaled-down Epstein frames (denoted by $P_{n}$ and $P_{s}$, respectively) and the corresponding total mass of the uniform zone, $m_{d}$; implying that the Epstein corners have no effect on $P_{\text {loss }}$, as expressed by 


$$
P_{l o s s 1}=\frac{P_{n}-P_{s}}{m_{d}}
$$

$P_{\text {loss }}$ is the averaged specific magnetization loss in the four corners of the standard Epstein frame, as shown in Fig.2(a). The total magnetization loss and volume (or mass) of the four corner sub-regions of E-25 are equivalent to those of the scaled-down Epstein E-17.5 or E-20, according to the assumptions given in sub-Section A. Therefore, the averaged specific magnetization loss in the non-uniform region of E-25, $P_{\text {loss } 2}$, can be determined from the absolute total power loss, measured from E-17.5 or E-20, denoted by $P_{s}$, and the corresponding active mass, $m_{a}$, of the total non-uniform regions of E-25, shown in Fig.2(a), i.e.,

$$
P_{\text {loss } 2}=\frac{P_{s}}{m_{a}}
$$

In order to obtain a closer approximation of the mean magnetic path length of the standard Epstein frame, $l_{e}$, a weighted processing method, based on the already obtained $l_{m l}$ and $l_{m 2}$, is proposed, i.e., $l_{e}$ becomes a weighted sum of $l_{m l}$ and $l_{m 2}$, incorporating the corresponding weight factors $\alpha$ and $\beta$, as given by

$$
l_{e}=\alpha \cdot l_{m 1}+\beta \cdot l_{m 2}
$$

where $\alpha$ and $\beta$ represent the contribution of $\frac{1}{P_{\text {loss }}}$ to the weighted $l_{e}$ from (1). Let

$$
\left\{\begin{array}{l}
P_{x}=\frac{1}{P_{\text {loss } 1}} \\
P_{y}=\frac{1}{P_{\text {loss } 2}}
\end{array}\right.
$$

then, the weighted factors $\alpha$ and $\beta$ are given by

$$
\left\{\begin{array}{l}
\alpha=\frac{P_{x}}{P_{x}+P_{y}} \\
\beta=\frac{P_{y}}{P_{x}+P_{y}}
\end{array}\right.
$$

To distinguish this from the next weighted processing stage described in the following sub-Section, (4)-(6) are referred to as the first-level weighted processing.

\section{2) Second-Level Weighted Processing}

The first step in the double Epstein frame method and the first-level weighted processing is to measure the total absolute losses (W) from the standard (E-25) and the scaled-down Epstein (E-17.5 or E-20) frames, denoted by $P_{n}$ and $P_{s}$ respectively, Next the difference between $P_{n}$ and $P_{s},(\Delta P)$ is expressed as

$$
\Delta P=P_{n}-P_{s}
$$

Note that for the different Epstein groups, $2 \mathrm{E}(25-17.5)$ and $2 \mathrm{E}(25-20)$, different forms of $\Delta P$ apply, i.e.,

$$
\left\{\begin{array}{lll}
\Delta P_{1}=P_{n 1}-P_{s 1} & \text { for } & 2 E(25-17.5) \\
\Delta P_{2}=P_{n 2}-P_{s 2} & \text { for } & 2 E(25-20)
\end{array}\right.
$$

Naturally, the following questions arise: (i) what is the difference in determining magnetic properties when using different Epstein groups (2E(25-17.5) or $2 \mathrm{E}(25-20))$ ? (ii) is it necessary, or possible, to further qualify the magnetic property modeling results by using a further level of weighted processing of the results obtained from the different Epstein groups?

In order to further investigate the effect of different Epstein groups on the weighted mean magnetic path length of the standard Epstein frame obtained from the first-level weighted processing, second-level weight factors, $\alpha \alpha$ and $\beta \beta$, are proposed, based on the difference of the absolute losses, measured from the two Epstein groups, $\Delta P_{I}$ (corresponding to $2 \mathrm{E}(25-17.5))$ and $\Delta P_{2}$ (corresponding to $2 \mathrm{E}(25-20)$ ). These are given by

$$
\left\{\begin{array}{l}
\alpha \alpha=\frac{\Delta P_{2}}{\Delta P_{1}+\Delta P_{2}} \\
\beta \beta=\frac{\Delta P_{1}}{\Delta P_{1}+\Delta P_{2}}
\end{array}\right.
$$

The weight factors, $\alpha \alpha$ and $\beta \beta$, defined in (9), imply that if the $\Delta P$ for one Epstein group is smaller compared to the other, then the field distributions over the corner-regions of the standard and scaled-down Epstein frames of that group will be more similar, the uniform region will be more uniform, and the value of the corresponding weight factor will be larger.

Once the first-level weighted processing, based on the Epstein group, is applied, the mean magnetic path length of the $25 \mathrm{~cm}$ Epstein frame can be obtained from 2E(25-17.5) and $2 \mathrm{E}(25-20)$, referred to as $l_{e 1}$ and $l_{e 2}$ respectively, and the second-level weighted mean magnetic path length of the $25 \mathrm{~cm}$ Epstein frame $l_{e l 2}$ can be determined from $l_{e l}$ and $l_{e 2}$, using

$$
l_{e 12}=\alpha \alpha \cdot l_{e 1}+\beta \beta \cdot l_{e 2}
$$

From (4) here $l_{e l}$ for the Epstein group 2E(25-17.5) and $l_{e 2}$ for Epstein group 2E(25-20) can be expressed as

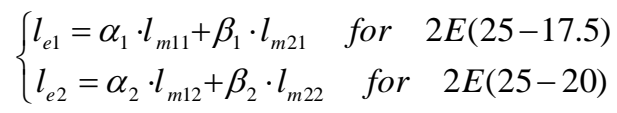

where

$\alpha_{1}, \beta_{1}$ : first-level weighted factors for $2 \mathrm{E}(25-17.5)$;

$l_{m 11}$ : mean magnetic path length for $2 \mathrm{E}(25-17.5)$ by $P_{\text {loss }}$;

$l_{m 21}$ : mean magnetic path length for $2 \mathrm{E}(25-17.5)$ by $P_{\text {loss } 2}$;

$\alpha_{2}, \beta_{2}$ : first-level weighted factors for $2 \mathrm{E}(25-20)$;

$l_{m 12}$ : mean magnetic path length for $2 \mathrm{E}(25-20)$ by $P_{\text {loss }}$;

$l_{m 22}$ : mean magnetic path length for $2 \mathrm{E}(25-20)$ by $P_{\text {loss } 2}$.

Note that the second-level weighted processing can be used to deal with other magnetic properties, such as specific magnetization loss and exciting power, when using two Epstein groups. 


\section{RESULTS AND DISCUSSION}

In order to show the benefits of the Epstein set, validate the proposed two-level weighted processing methods and demonstrate the variation of magnetic properties of GO electrical steel with test conditions, an extensive set of Epstein measurements has been carried out over a range of excitation frequencies, strip angles, and ambient temperatures.

The experimental results, as presented in sub-Sections $B, C$ and $D$ of this Section, were measured at a normal laboratory ambient temperature of $25^{\circ} \mathrm{C}$. All Epstein strips were $300 \mathrm{~mm}$ long so when inserted into the two smaller frames, their ends overhung as shown in Fig.2(b). The maximum normal flux density at these overhangs was less than $0.50 \mathrm{mT}$ (measured using an Gauss/Teslameter, Model 7010), while $B_{m}$ (the maximum magnetic flux density, $B$ ) inside the limb reached 1.9T. The eddy currents induced by normal flux in the overhang regions were negligible.

\section{A. Multi-Angle Sampling to the RD}

Epstein strips $(300 \mathrm{~mm} \times 30 \mathrm{~mm})$ were cut at eight angles $\theta$ to the RD from wide sheets from locations illustrated in Fig.3 for investigating the effect of magnetizing direction on the measured magnetic properties of the steel. Two grades of $0.30 \mathrm{~mm}$ thick GO electrical steel, 30P120, POSCO, Korea, and 30Q120, WISCO, China, were used in this multi-angle testing.

Clearly, the multi-angle testing is still a case of onedimensional magnetic measurement and modeling, but it should be noted that the measured loss in strips cut at angles other than $0^{\circ}$ and $90^{\circ}$ to the RD depend on the way in which the strips are stacked in the frame [24]. Furthermore, it should be noted that the vector distributions of both magnetic fields and flux density vary with the strip width (such as $25 \mathrm{~mm}$ and $100 \mathrm{~mm}$ reported in [25]). This undoubtedly affects the actual mean magnetic path length. In this investigation, only $30 \mathrm{~mm}$ wide strips were considered.

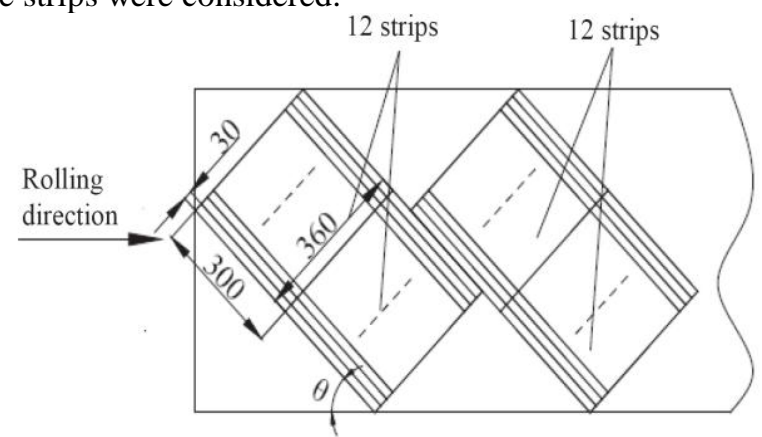

Fig.3. Schematic diagram showing position of strips cut from $100 \mathrm{~cm}$ wide sheet at angles $\theta$ to the RD.

\section{B. Specific Magnetization Loss at Room Temperature}

The specific magnetization losses of strips of 30P120 cut at different angles to the RD, using the Epstein groups $2 \mathrm{E}(25$ $17.5)$ and $2 \mathrm{E}(25-20)$, and the standard Epstein frame (E-25) magnetized at $50 \mathrm{~Hz}$, are shown in Fig.4. Each data point on the graphs is the average of the measurements; all of which fell within the nominal $1 \%$ repeatability of the test equipment. Significant points to note are:
1) The identical results shown in Fig.4(a) from the three setups (results obtained by standard Epstein and two Epstein groups) demonstrate that the approach is valid for measuring the specific magnetization loss of strips cut parallel to the RD. It implies that the assumed $0.94 \mathrm{~m}$ mean magnetic path length in the standard $25 \mathrm{~cm}$ frame is sufficiently accurate under these specific test conditions.

2) Fig.4(b) shows that the results obtained from $2 \mathrm{E}(25-17.5)$ and $2 \mathrm{E}(25-20)$ at strip angle $55^{\circ}$ agree to within $2 \%$ over the full flux density range, but, they are up to $21 \%$ higher than the results obtained from the Epstein $(25 \mathrm{~cm})$ frame alone.

3) Fig.4(c) shows that the specific magnetization losses measured using the two Epstein groups at strip angle $90^{\circ}$, differ by up to $22 \%$, but, they both are up to $40 \%$ higher than those obtained from the standard Epstein frame.

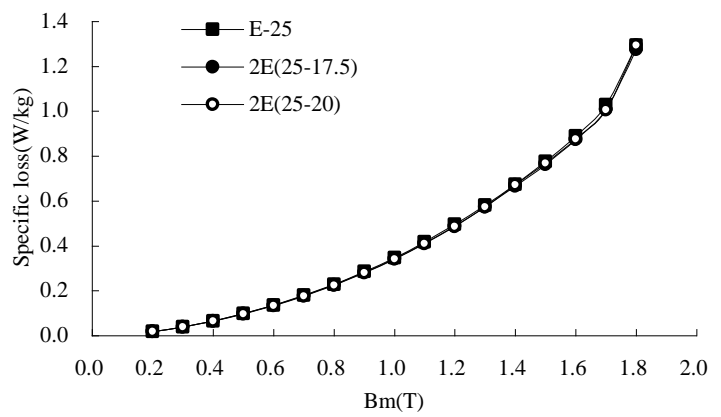

(a) Strip angle $0^{\circ}$

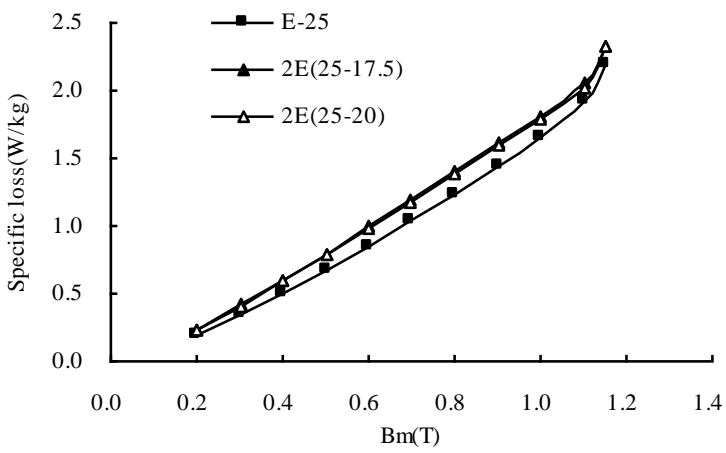

(b) Strip angle $55^{\circ}$

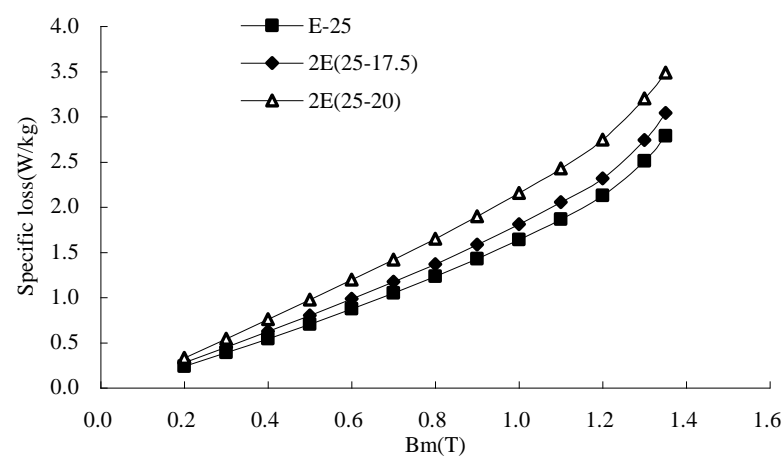

(c) Strip angle $90^{\circ}$

Fig.4. Variation of specific magnetization losses with flux density $(50 \mathrm{~Hz})$ measured in strips $30 \mathrm{P} 120$ cut at $0^{\circ}, 55^{\circ}$ and $90^{\circ}$ to the RD.

The results shown in Fig.4(b) suggest that the Epstein group must be used to obtain a more accurate measurement of loss at a strip angle $55^{\circ}$; but, there is no improvement in the results at 
a strip angle $90^{\circ}$. This implies that the assumption of identical magnetic field and loss over the non-uniform regions at a strip angle of $90^{\circ}$, is not valid and the results are sensitive to the size of the uniform region. As mentioned above, in order to improve the accuracy of the specific magnetization losses, as shown in Fig.4.(c), the use of a second-level weighted processing, based on the specific magnetization losses determined by Epstein groups (2E(25-17.5) and $2 \mathrm{E}(25-20))$, is recommended.

\section{Exciting Power at Room Temperature}

The variation of exciting power with flux density of the same 30P120 strips of GO electrical steel, cut at various angles to the RD, was measured in the Epstein frame (E-25) and the two Epstein groups (2E(25-17.5) and $2 \mathrm{E}(25-20)$ ) at an excitation frequency of $50 \mathrm{~Hz}$. The exciting power was calculated from readings obtained from a YOKOGAWA WT3000 power analyzer (with calibrated uncertainty of $1 \times 10^{-4}$ given by NIM), shown connected to the $25 \mathrm{~cm}$ Epstein frame in Fig.5. This was compared with the existing Epstein system for further confirmation of the measured exciting power [21].

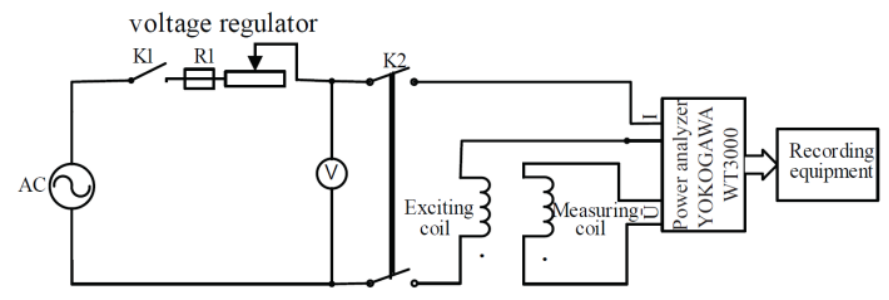

Fig.5. Experimental set up for measuring exciting power by means of a power analyzer (WT-3000).

The trends in the variation of exciting power, $P_{e}$, with flux density in strips cut at $0^{\circ}, 55^{\circ}$ and $90^{\circ}$ to the RD are shown in Fig. 6 and can be summarized as:

1) The results shown in Fig.6(a) and (b) demonstrate that no significant difference in the values of the exciting power for strip angles $0^{\circ}$ and $55^{\circ}$ was found between the results from $2 \mathrm{E}(25-17.5), 2 \mathrm{E}(25-20)$, the power analyzer (WT-3000) and the standard Epstein frame (E-25).

2) Fig.6(c) shows that, although the results obtained by $2 \mathrm{E}(25-17.5)$ and $2 \mathrm{E}(25-20)$ at strip angle $90^{\circ}$ agree closely, they differ significantly from those obtained by the standard Epstein frame (E-25), suggesting that the Epstein group method used at strip angle $90^{\circ}$ would provide an improved measurement of $P_{e}$.

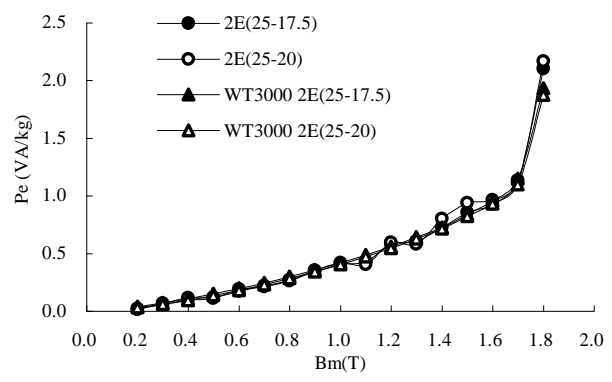

(a) Strip angle $0^{\circ}$

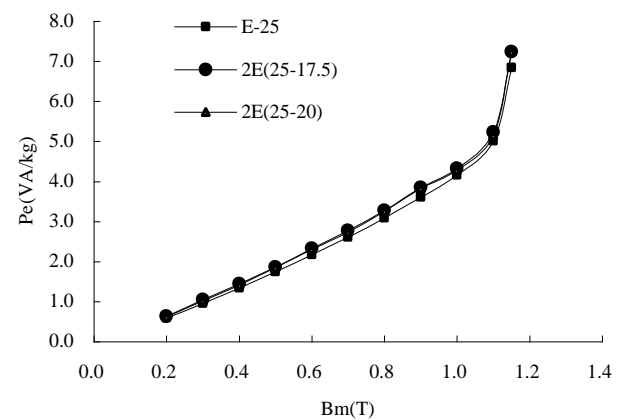

(b) Strip angle $55^{\circ}$

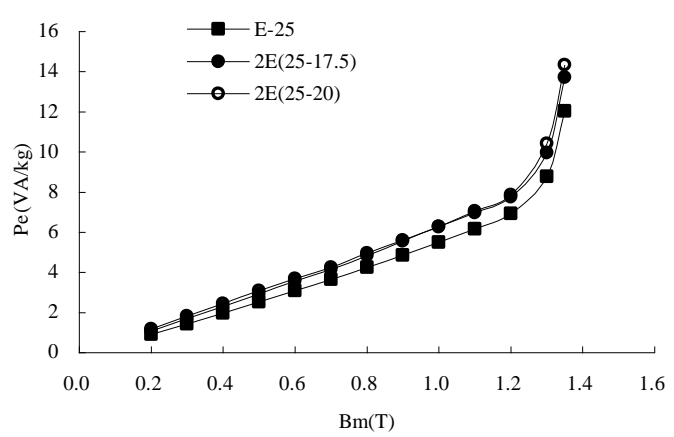

(c) Strip angle $90^{\circ}$

Fig.6. Variation of exciting power with flux density in strips of 30P120 cut at $0^{\circ}, 55^{\circ}$, and $90^{\circ}$ to the $\mathrm{RD},(50 \mathrm{~Hz})$.

\section{Mean Magnetic Path Length of the Epstein Frame Measured at Room Temperature}

The weighted mean magnetic path length of the standard Epstein frame (E-25) $l_{e}$ (referred to as the first-level weighted processing result), $l_{m l}$ (non-weighted and based on the specific magnetization loss inside the uniform zone, $\left.P_{\text {loss }}\right)$, and $l_{m 2}$ (non-weighted and based on the specific magnetization loss inside the non-uniform zone, $P_{\text {loss } 2}$ ) were determined for the $30 \mathrm{P} 120$ grade steel, using Epstein group 2E(25-17.5) at $50 \mathrm{~Hz}$, as shown in Fig.7.

The trends in the variation of the path lengths with magnetic flux densities and strip angles can be summarized as:

1) While $l_{m 1}$ and $l_{m 2}$ of the Epstein frame $(25 \mathrm{~cm})$ are different, the weighted mean magnetic path length, $l_{e}$, of the Epstein frame lies between them. It can be seen from Fig.7(a) that $l_{e}$ varies within a narrow range, i.e., from $0.940 \mathrm{~m}$ to $0.945 \mathrm{~m}$, and is not a constant value when the flux density increases, for strips cut parallel to the RD.

2) The mean magnetic path length of the standard Epstein frame (E-25) is not always $0.94 \mathrm{~m}$ as specified in the IEC standard, e.g., as shown in Fig.7(b) and (c). It is approximately $0.93 \mathrm{~m}$ for the strip angle $55^{\circ}$ and $0.92 \mathrm{~m}$ for a strip angle $90^{\circ}$.

In order to further investigate the effect of excitation frequency and strip angle on the Epstein mean magnetic path length, two forms of weighted processing methods, giving results referred to as $l_{e 1}$ and $l_{e 2}$ for first-level weight, and $l_{e 12}$ for second-level weight, were applied. The weighted mean magnetic path lengths related to the standard Epstein frame (E25), using two-level weighted processing, with different excitation frequencies and strip angles are also summarized in Table I to numerically show the varying trends. It should be 
noted that the uncertainty in the value of the mean magnetic path length is approximately $\pm 1 \%$.

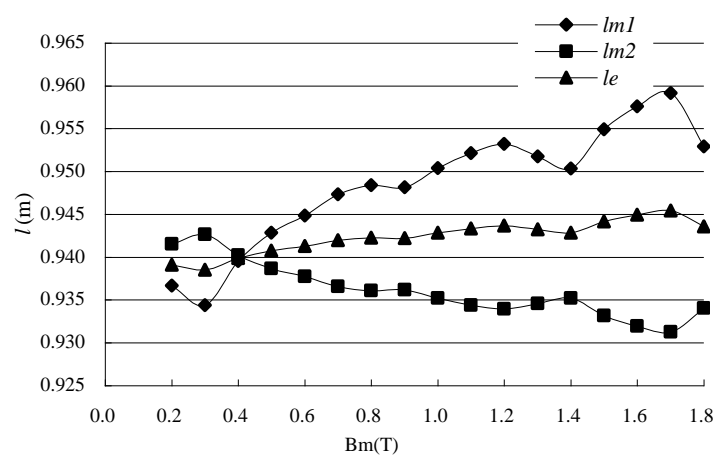

(a) Strip angle $0^{\circ}$

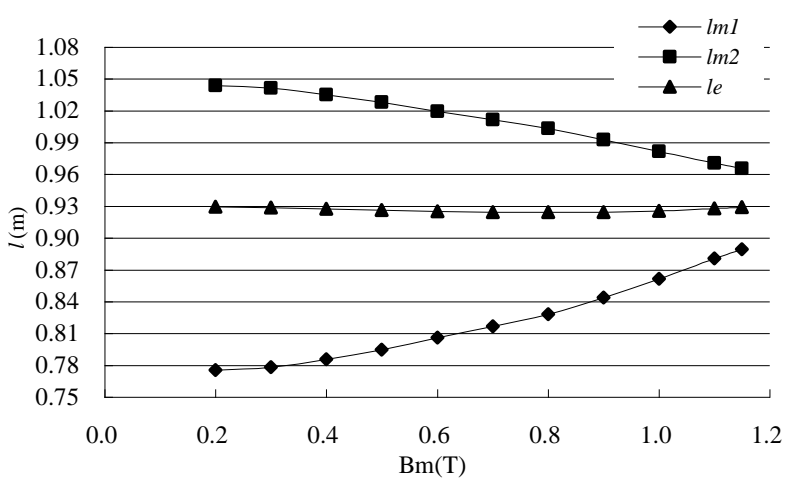

(b) Strip angle $55^{\circ}$

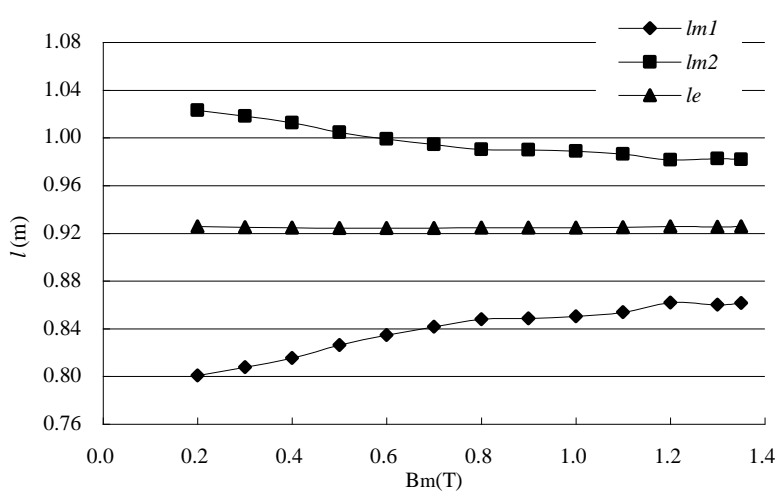

(c) Strip angle $90^{\circ}$

Fig.7. Variation of mean magnetic path length of the Epstein frame $(25 \mathrm{~cm})$ with flux density measured using $2 \mathrm{E}(25-17.5)$, at $50 \mathrm{~Hz}, 30 \mathrm{P} 120$.

TABLE I

Weighted MEAN MAGNeTic PATH LeNGTHS OF EPSTEIN FRAME (25CM)

\begin{tabular}{|c|c|c|c|c|c|c|}
\hline \multirow{2}{*}{$\begin{array}{c}\text { Strip angles to } \\
\mathrm{RD}\end{array}$} & \multicolumn{5}{|c|}{ Excitation frequency } \\
\cline { 2 - 7 } & \multicolumn{3}{|c|}{$50(\mathrm{~Hz})$} & \multicolumn{3}{|c|}{$300(\mathrm{~Hz})$} \\
\cline { 2 - 7 } & $l_{e l}(\mathrm{~m})$ & $l_{e 2}(\mathrm{~m})$ & $l_{e l 2}(\mathrm{~m})$ & $l_{e l}(\mathrm{~m})$ & $l_{e 2}(\mathrm{~m})$ & $l_{e l 2}(\mathrm{~m})$ \\
\hline $0^{\circ}$ & $0.94^{+}$ & $0.94^{-}$ & 0.94 & $0.94^{+}$ & $0.94^{-}$ & 0.94 \\
\hline $55^{\circ}$ & $0.93^{-}$ & $0.90^{+}$ & $0.91^{+}$ & $0.94^{-}$ & $0.90^{+}$ & 0.92 \\
\hline $90^{\circ}$ & $0.93^{-}$ & 0.90 & $0.91^{+}$ & $0.93^{-}$ & $0.90^{+}$ & $0.91^{+}$ \\
\hline
\end{tabular}

Notes:

(1) $l_{e l}(\mathrm{~m})$ : first-level weighted mean magnetic path length, by $2 \mathrm{E}(25-17.5)$;

(2) $l_{e 2}(\mathrm{~m})$ : first-level weighted mean magnetic path length, by $2 \mathrm{E}(25-20)$;
(3) $l_{e l 2}(\mathrm{~m})$ : second-level weighted mean magnetic path length, by $2 \mathrm{E}(25-17.5)$ and $2 \mathrm{E}(25-20)$

(4) The weighted mean magnetic path length is not a constant value; in this Table, the number with "+" means "greater than", and "-" means "less than".

\section{E. Epstein Tests at Higher Controlled Temperatures}

In practice, many transformer cores, for example, some drytype transformers, potentially operate at temperatures up to $125^{\circ} \mathrm{C}$. Therefore it is important to be aware of any influence of temperature on the measurement equipment or the basic properties of the GO steel which would cause additional variation in the mean magnetic path length over this range.

The Epstein group 2E(25-17.5) was investigated with the measurement rigs placed in a temperature controlled chamber (Test Chamber, GDJS-010L) with non-magnetic wall, as shown in Fig.8. The temperature was kept to within $\pm 0.5^{\circ} \mathrm{C}$ over the range from $25^{\circ} \mathrm{C}$ to $125^{\circ} \mathrm{C}$. The effect of varying ambient temperature on the mean magnetic path length was studied on strips of $30 \mathrm{Q} 120$ cut at strip angles $0^{\circ}$ and $90^{\circ}$ to the $\mathrm{RD}$, at $50 \mathrm{~Hz}$ excitation frequency.

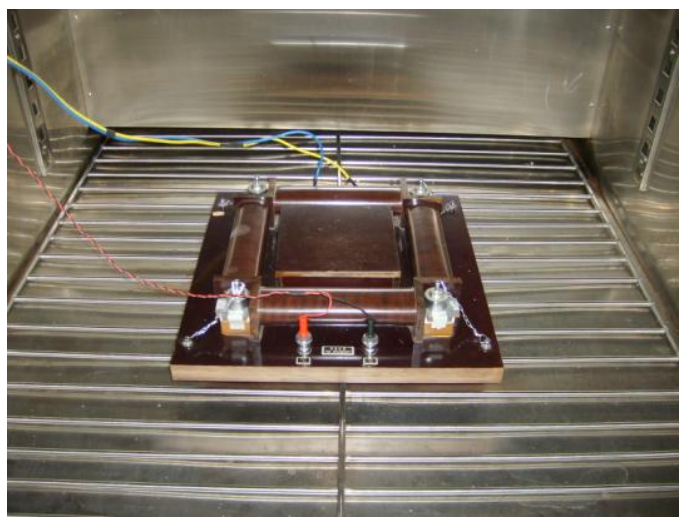

Fig.8. Epstein frame located in the chamber for measurements under controlled temperature conditions.

\section{(1)Variation of Specific Magnetization Loss with Temperature}

The specific magnetization losses of strips of $30 \mathrm{Q} 120$, cut at $0^{\circ}$ to the $\mathrm{RD}$, were measured using the Epstein group $2 \mathrm{E}(25-$ 17.5), at ambient temperatures ranging from $25^{\circ} \mathrm{C}$ to $125^{\circ} \mathrm{C}$ at $50 \mathrm{~Hz}$.

Table II demonstrates that the specific magnetization losses vary with flux density and ambient temperatures within the above mentioned ranges.

The relative deviation, $p p_{y}$, of the specific magnetization loss, $P_{\text {loss }(y)}$ at a given ambient temperature (y, ranging from $25^{\circ} \mathrm{C}$ to $125^{\circ} \mathrm{C}$ ) with respect to that measured at $25^{\circ} \mathrm{C}, P_{\text {loss(25) }}$, is examined using (12)

$$
p p_{y}=\frac{P_{l o s s(y)}-P_{l o s s(25)}}{P_{l o s s(25)}} \times 100 \quad(\mathrm{y}=50,75,100,125)
$$

It is observed from Table II that at high temperatures, e.g. at $125^{\circ} \mathrm{C}$, and low flux density, the specific magnetization losses are lower, and the relative difference between the measured ambient and high temperature specific losses can be up to 7 
$8 \%$; for example, $P P_{y}$ is $-8 \%$ when flux density is $0.4 \mathrm{~T}$ and temperature is $125^{\circ} \mathrm{C}$.

TABLE II

VARIATION OF SPECIFIC TOTAL LOSSES WITH FLUX DENSITY AT DIFFERENT AMBIENT TEMPERATURES

\begin{tabular}{|c|c|c|c|c|c|}
\hline \multirow{2}{*}{$\begin{array}{c}\text { Flux } \\
\text { Densities }\end{array}$} & \multicolumn{5}{|c}{ Specific total losses at ambient temperatures $(\mathrm{W} / \mathrm{kg})$} \\
\cline { 2 - 6 } & $25\left({ }^{\circ} \mathrm{C}\right)$ & $50\left({ }^{\circ} \mathrm{C}\right)$ & $75\left({ }^{\circ} \mathrm{C}\right)$ & $100\left({ }^{\circ} \mathrm{C}\right)$ & $125\left({ }^{\circ} \mathrm{C}\right)$ \\
\hline 0.10 & 0.004 & 0.004 & 0.004 & 0.004 & 0.004 \\
\hline 0.20 & 0.016 & 0.016 & 0.016 & 0.015 & 0.015 \\
\hline 0.30 & 0.036 & 0.035 & 0.034 & 0.033 & 0.033 \\
\hline 0.40 & 0.062 & 0.061 & 0.059 & 0.058 & 0.057 \\
\hline 0.50 & 0.094 & 0.092 & 0.090 & 0.088 & 0.087 \\
\hline 0.60 & 0.131 & 0.129 & 0.126 & 0.124 & 0.122 \\
\hline 0.70 & 0.174 & 0.172 & 0.168 & 0.165 & 0.162 \\
\hline 0.80 & 0.224 & 0.220 & 0.216 & 0.212 & 0.208 \\
\hline 0.90 & 0.280 & 0.276 & 0.270 & 0.265 & 0.261 \\
\hline 1.00 & 0.343 & 0.337 & 0.331 & 0.325 & 0.319 \\
\hline 1.10 & 0.413 & 0.407 & 0.399 & 0.391 & 0.385 \\
\hline 1.20 & 0.491 & 0.483 & 0.474 & 0.466 & 0.457 \\
\hline 1.30 & 0.576 & 0.567 & 0.557 & 0.547 & 0.537 \\
\hline 1.40 & 0.670 & 0.660 & 0.649 & 0.637 & 0.626 \\
\hline 1.50 & 0.772 & 0.762 & 0.750 & 0.737 & 0.725 \\
\hline 1.55 & 0.828 & 0.818 & 0.805 & 0.793 & 0.780 \\
\hline 1.60 & 0.891 & 0.881 & 0.867 & 0.855 & 0.842 \\
\hline 1.65 & 0.962 & 0.952 & 0.939 & 0.927 & 0.916 \\
\hline 1.70 & 1.051 & 1.042 & 1.031 & 1.022 & 1.014 \\
\hline 1.75 & 1.174 & 1.167 & 1.157 & 1.151 & 1.146 \\
\hline 1.80 & 1.360 & 1.352 & 1.345 & 1.338 & 1.333 \\
\hline 1.85 & 1.590 & 1.582 & 1.571 & 1.558 & 1.554 \\
\hline
\end{tabular}

\section{(2) Variation of Exciting Power with Temperature}

The exciting power, $P_{e}$, for the strips of the $30 \mathrm{Q} 120$ grade steel, cut at $0^{\circ}, 55^{\circ}$ and $90^{\circ}$ to the $\mathrm{RD}$, was measured using the standard Epstein (E-25) and Epstein group 2E(25-17.5) at ambient temperature ranging from $25^{\circ} \mathrm{C}$ to $75^{\circ} \mathrm{C}$. The results shown in Fig.9 indicate that it is constant over this range for each strip angle, as was found in the case of the specific magnetization loss.

However, a considerable difference in magnitude is found when using the standard Epstein frame and the Epstein group $2 \mathrm{E}(25-17.5)$.

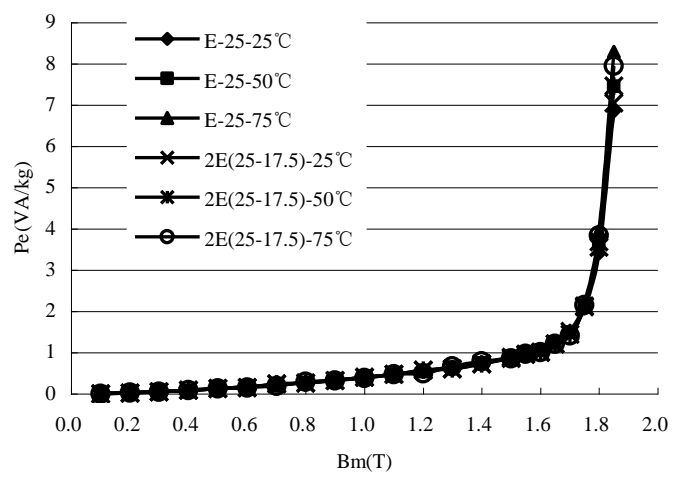

(a) Strip angle $0^{\circ}$

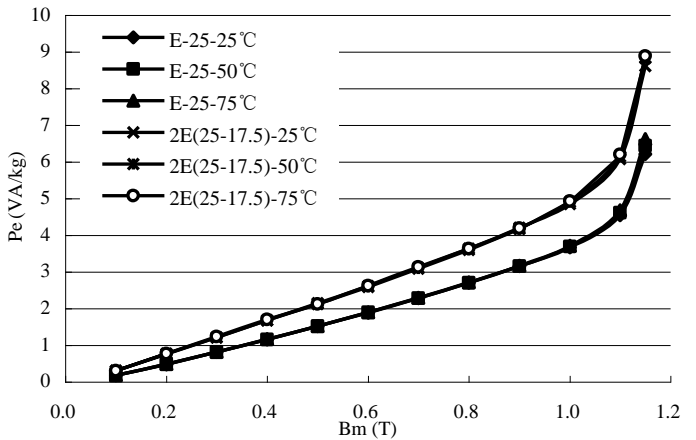

(b) Strip angle $55^{\circ}$

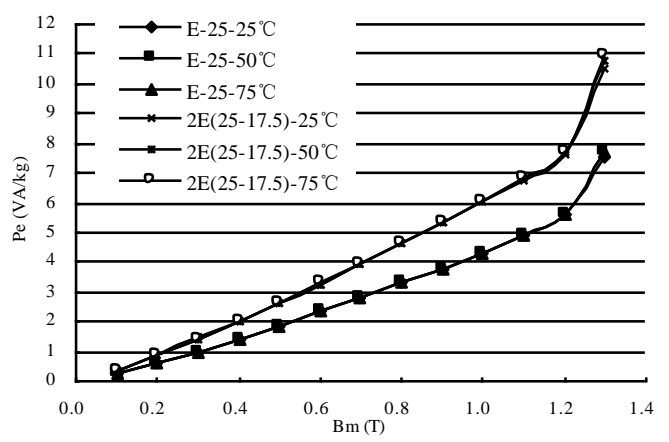

(c) Strip angle $90^{\circ}$

Fig. 9. Variation of exciting power with flux density measured at different temperatures using Epstein (E-25) and 2E(25-17.5) at different strip angles.

\section{(3)Variation of Mean Magnetic Path Length with Temperature}

The mean magnetic path lengths of the standard Epstein frame (E-25), at different magnetic flux density, different temperature (from $25^{\circ} \mathrm{C}$ up to $125^{\circ} \mathrm{C}$ at $25^{\circ} \mathrm{C}$ increments) and at different strip angle $\left(0^{\circ}, 55^{\circ}\right.$, and $\left.90^{\circ}\right)$ were also determined using Epstein group (2E(25-17.5), for strips 30Q120.

The relative decrease $\left(l l_{x}\right)$ of the weighted mean magnetic path lengths, $l_{e(x)}$, obtained at the elevated temperatures (from $50^{\circ} \mathrm{C}$ to $\left.125^{\circ} \mathrm{C}\right)$ with respect to that obtained at $25^{\circ} \mathrm{C}\left(l_{e(25)}\right)$ can be written as,

$$
l l_{x}=\frac{l_{e(x)}-l_{e(25)}}{l_{e(25)}} \times 100 \quad(x=50,75,100,125)
$$

Further examination, using (13), concludes that the mean magnetic path length of the Epstein frame (30Q120, strip angle: $0^{\circ}$ ) decreases with increasing temperature (from $25^{\circ} \mathrm{C}$ to $125^{\circ} \mathrm{C}$ ), and the maximum deviation is at most below $0.3 \%$.

\section{CONCLUSION}

The extended modeling of magnetic properties of GO electrical steel based on an Epstein combination and two-level weighted processing, and the related experimental results can be summarized as:

1) The double Epstein frame method, in fact based on an Epstein subtraction scheme (using 2E(25-17.5) or 2E(25-20)), eliminates the effect of the non-uniformity of the specific magnetization loss over the corner regions of the entire frame, and enables the accurate measurement of the specific 
magnetization loss and exciting power in the uniformly magnetized limb regions.

2) Two-level weighted processing methods based on the loss data, obtained by an Epstein set (E-25, E-20, and E-17.5), are proposed and implemented, offering two benefits: (i) the first-level weighted method takes the non-uniformity of the magnetic field and the power loss inside the entire Epstein frame into account, making it possible to accurately determine the mean magnetic path length of the Epstein frame; (ii) the second-level weighted method can be used to further examine the effect of different Epstein groups (2E(25-17.5) and 2E(2520)) on the mean magnetic path length, specific magnetization loss and exciting power.

3) All the measurements covering the many Epstein test cases, adequately demonstrate the Epstein combination and two-level weighted processing methods and can be safely carried out. The corresponding results also show the impacts of the related factors, such as magnetic flux density, non-RD magnetization, excitation frequency, and ambient temperature, on Epstein magnetic properties.

4) Measurements using the standard Epstein frame (E-25) are subject to errors because the value of magnetic path length is fixed. As demand for more accurate measurements under non-standard conditions increases, it is becoming more important to quantify, or even eliminate, these errors. While intended for academic interest, it is hoped that this work stimulates discussion and debate amongst the steel manufacturers, users and researchers to consider the methodologies proposed in this paper or other approaches, to address the unsatisfactory nature of the Epstein measurement for modern and future industrial needs.

\section{ACKNOWLEDGMENT}

The authors wish to thank Mr. Guisheng Han and Mr.Fulai Che of the Institute of Applied Electrotechnology of Tianwei Group, Baoding, for their help in Epstein experiments, and thank Dr. Shuo Liu for helpful technical discussion. This project was supported by the National Natural Science Foundation of China under Grant 51107026, by the China Postdoctoral Science Foundation under Grants 2011M500534, 2012T50242 and 2013M530866, and by the State Grid Corporation of China under Grant sgri-wd-71-13.

\section{REFERENCES}

[1] J.Sievert, "Recent advances in the one- and two-dimensional magnetic measurement technique for electrical steel sheet," IEEE Trans. on Magn., vol.26, no.5, pp.2553-2558, 1990.

[2] A.J.Moses, "Importance of rotational losses in rotating machines and transformer," J. Mat. Eng. and Performance, 1(2), pp.235-244, 1992.

[3] M.Nakano, H.Nishimoto, K.Fujiwara, et al, "Improvements of single sheet tester for measurement of 2-D magnetic properties up to high flux density," IEEE Trans. on Magn., vol.35, no.5, pp.3965-3967, 1999.

[4] K.Fujiwara, T.Adachi, and N.Takahashi, "A proposal of finite-element analysis considering two-dimensional magnetic properties," IEEE Trans. on Magn., vol.38, no.2, pp.889-892, 2002.

[5] J.Zhu, J.Zhong, Z.Lin, and J.Sievert, "Measurement of magnetic properties using 3-D magnetic excitations," IEEE Trans. on Magn., vol.39, no.5, pp.3429-3431, 2003.
[6] M.Enokizono, H.Shimoji, A.Ikariga, et al, "Vector magnetic characteristic analysis of electrical machines," IEEE Trans. on Magn., vol.41, no.5, pp.2032-2035, 2005.

[7] Y.Li, J.Zhu, Q.Yang, et al, "Measurement of soft magnetic composite material using an improved 3-D tester with flexible excitation coil and novel sensing coils," IEEE Trans. on Magn., vo.46, no.6, pp.1971-1974, 2010.

[8] T.Yamamoto and Y.Ohya, "Single sheet tester for measuring core losses and permeabilities in a silicon steel sheet," IEEE Trans. on Magn., vol.10, no.2, pp.157-159, 1974.

[9] A.J.Moses and S.Hamadeh, "Comparison of the Epstein frame and single strip tester for measuring the power loss of non-oriented electrical steels, IEEE Trans. on Magn., Vol.19, no.6, pp. 2705-2710, 1983.

[10] IEC 60404-2 AMD 1-2008: Magnetic Materials - Part 2: Methods of measurement of the magnetic properties of electrical steel sheet and strip by means of an Epstein frame; Amendment 1.

[11] IEC 60404-3-2010: Magnetic Materials - Part 3: Methods of measurement of the magnetic properties of electrical steel strip and sheet by means of a single sheet tester - Edition 2.2; Consolidated Reprint.

[12] Z.Cheng, N.Takahashi, B.Forghani, et al, "Effect of variation of B-H properties on loss and flux inside silicon steel lamination," IEEE Trans. on Magn., vol.47, no.5, pp.1346-1349, 2011.

[13] X.Zhao, J.Lu, L.Li, Z.Cheng, and T.Lu, "Analysis of the DC bias phenomenon by the harmonic balance finite element method," IEEE Trans. on Power Delivery, vol.26. no.1, pp.475-485, 2011.

[14] T.L.Mthombeni, P.Pillay, and R.M.W.Strnat, "New Epstein frame for lamination core loss measurements under high frequencies and high flux densities," IEEE Trans on Energy Conversion, vol.22, no.3, pp.614-620, 2007.

[15] C.Patsios, E.Tsampouris, M.Beniakar, and A.Kladas, "Dynamic finite element hysteresis model for iron loss calculation under PWM excitation," IEEE Trans. on Magn., vol.47, no.5, pp.1130-1133, 2011.

[16] A.J.Moses, "Characterisation and performance of electrical steels for power transformers operating under extremes of magnetization conditions,"(invited) International Colloquium Transformer Research and Asset Management, Croatia, Nov. 12-14, 2009.

[17] IEC TR 62383-2006 Determination of magnetic loss under magnetic polarization waveforms including higher harmonic components. Measurement, modeling and calculation methods.

[18]P.Marketos, S.Zurek, and A.J.Moses, "Calculation of the mean path length of the Epstein frame under non-sinusoidal excitations using the double Epstein method," J. Magn. Mag. Mat., (2008) 320 2542-2545.

[19] Z.Cheng, N.Takahashi, B.Forghani, et al., "Engineering-oriented benchmarking and application-based magnetic material modeling in transformer research," (invited) International Colloquium Transformer Research and Asset Management, Croatia, May 16-18, 2012.

[20] Z.Cheng, N.Takahashi1,B.Forghani, L.Liu, Y.Fan, T.Liu, J.Zhang, and X.Wang, "3-D finite element modeling and validation of power frequency multi-shielding effect," IEEE Trans. on Magn., vol.48, no.2, pp.243-246, 2012.

[21] Z.Cheng, N.Takahashi, B.Forghani, et al. Electromagnetic and Thermal Field Modeling and Application in Electrical Engineering. Science Press, Beijing, 2009.

[22] IEEE Std 1597.1 ${ }^{\mathrm{TM}}$-2008: IEEE Standard for Validation of Computational Electromagnetics Computer Modeling and Simulations.

[23] TEAM Benchmark Problems. [Online] available: www.compumag.org /TEAM.

[24] D.Davies and A.J.Moses, "Causes of some error in measurements in grain-oriented silicon-iron using the Epstein square," Journal of Magnetism and Magnetic Materials, 26 (1982) 35-36.

[25] N.J.Layland, A.J.Moses, N.Takahashi, et al., "Effects of shape on samples of silicon-iron on the directions of magnetic field and flux density," Nonlinear Electromagnetic System. (Eds.) A.J.Moses and A.Basak, IOS Press, pp.800-803, 1996. 


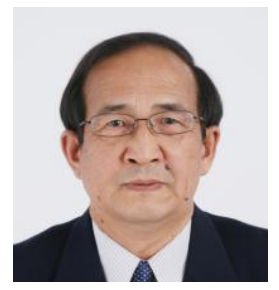

Zhiguang Cheng was born in 1942, China. He graduated from Peking University in 1967 and received a $\mathrm{Ph} . \mathrm{D}$. degree in Electrical Engineering from Tsinghua University in 1995 . He is currently a deputy chief engineer, senior engineer, and head of Electromagnetic Laboratory of the R \& D Center, Baoding Tianwei Group Co. He has received the National and Ministerial Science and Technology awards for his contributions to engineering science research and application. He is a founding member of the international Compumag Society, a senior member of IEEE, and a senior member of the Chinese Electrotechnical Society. His primary research interests include engineering electromagnetic field analysis and validation, magnetic material modeling and industrial applications.

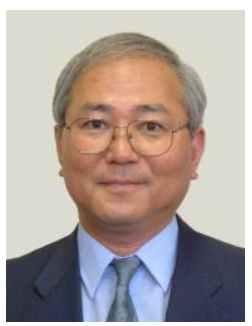

Norio Takahashi was born in 1951, Japan. He received a B.E. degree from Okayama University in 1974 and M.E. and Ph.D. degrees from Kyoto University in 1976 and 1982, respectively. He was a professor of Department of Electrical and Electronic Engineering, Chair of Electromagnetic Device Laboratory of Okayama University, Vice President of Power and Energy Society, IEE of Japan, Vice President of International Compumag Society, and IEEE Fellow. His major interests

were the development of numerical methods for calculating magnetic fields and optimal design methods for magnetic devices.

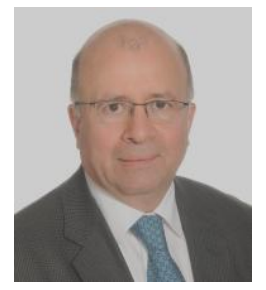

Behzad Forghani was born in 1957, Iran. He received a B. Eng. degree in 1980 and a M. Eng. degree in 1981, both in Electrical Engineering, from McGill University in Montreal, Canada. Since 1981, he has been working at Infolytica Corporation in the field of Computational Electromagnetics and is currently a Vice President. He has been an IEEE member since January 1980 and is a member of OIQ (Order of Engineers in Quebec). He regularly serves as a reviewer of Compumag and CEFC (two conferences with focus on the electromagnetic field computation) papers and is a Board Member of the International Compumag Society. His areas of interest are numerical techniques, material modeling, applications/devices and coupled problems.

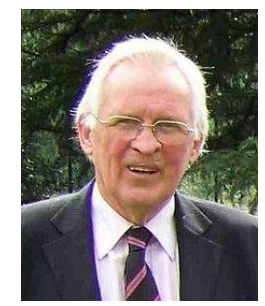

Anthony John Moses (M'87) was born in Newport, Gwent UK. He received his B.Eng. Tech. and $\mathrm{PhD}$ from the University of Wales in 1966 and 1990 followed by a DSc in 1990 for his contribution to research into the properties and applications of soft magnetic materials. He was appointed Professor of Magnetics and Director of the Wolfson Centre for Magnetics at Cardiff University in1992. He is author of over 500 publications on production, characterisation and applications of magnetic materials and previously was chairman of the UK Magnetics Society and the International organising committee of the Soft Magnetic Materials (SMM) series of conferences. He is a member of organising and editorial committees of several other international conferences and journals. He retired from his post at the university in 2012 and is now an Emeritus Professor at Cardiff University where he continues his research and consultancy interests at the Wolfson Centre.

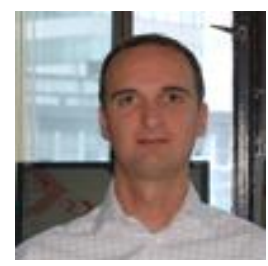

Philip Anderson was born in Wales, UK in 1972. $\mathrm{He}$ received a BEng and MSc from Cardiff University and following this worked with European Electrical Steels in Newport. He received his $\mathrm{PhD}$ from Cardiff University in 2000 and worked at the Wolfson Centre, Cardiff University since this time as a researcher and then Lecturer in Magnetic Engineering. He is a Chartered Engineer and active member of national and international committees on magnetic steels and alloys. 